\title{
Nanostructural Organization of Naturally Occurring Composites-Part I: Silica-Collagen-Based Biocomposites
}

\author{
Hermann Ehrlich, ${ }^{1}$ Sascha Heinemann, ${ }^{1}$ Christiane Heinemann, ${ }^{1}$ Paul Simon, ${ }^{2}$ \\ Vasily V. Bazhenov, ${ }^{3}$ Nikolay P. Shapkin, ${ }^{3}$ René Born, ${ }^{1}$ Konstantin R. Tabachnick, ${ }^{4}$ \\ Thomas Hanke, ${ }^{1}$ and Hartmut Worch ${ }^{1}$ \\ ${ }^{1}$ Max Bergmann Center of Biomaterials and Institute of Materials Science, Dresden University of Technology, \\ 01069 Dresden, Germany \\ ${ }^{2}$ Max Planck Institute of Chemical Physics of Solids, 01187 Dresden, Germany \\ ${ }^{3}$ Institute of Chemistry and Applied Ecology, Far Eastern National University, 690650 Vladivostok, Russia \\ ${ }^{4}$ P.P. Shirshov Institute of Oceanology, Russian Academy of Sciences, Nahimovsky pr. 36, 117997 Moscow, Russia
}

Correspondence should be addressed to Hermann Ehrlich, hermann.ehrlich@tu-dresden.de

Received 2 November 2007; Accepted 31 December 2007

Recommended by Donglu Shi

\begin{abstract}
Glass sponges, as examples of natural biocomposites, inspire investigations aiming at both a better understanding of biomineralization mechanisms and novel developments in the synthesis of nanostructured biomimetic materials. Different representatives of marine glass sponges of the class Hexactinellida (Porifera) are remarkable because of their highly flexible basal anchoring spicules. Therefore, investigations of the biochemical compositions and the micro- and nanostructure of the spicules as examples of naturally structured biomaterials are of fundamental scientific relevance. Here we present a detailed study of the structural and biochemical properties of the basal spicules of the marine glass sponge Monorhaphis chuni. The results show unambiguously that in this glass sponge a fibrillar protein of collagenous nature is the template for the silica mineralization in all silica-containing structural layers of the spicule. The structural similarity and homology of collagens derived from M. chuni spicules to other sponge and vertebrate collagens have been confirmed by us using FTIR, amino acid analysis and mass spectrometric sequencing techniques. We suggest that nanomorphology of silica formed on proteinous structures could be determined as an example of biodirected epitaxial nanodistribution of amorphous silica phase on oriented fibrillar collagen templates. Finally, the present work includes a discussion relating to silica-collagen-based hybrid materials for practical applications as biomaterials.
\end{abstract}

Copyright (c) 2008 Hermann Ehrlich et al. This is an open access article distributed under the Creative Commons Attribution License, which permits unrestricted use, distribution, and reproduction in any medium, provided the original work is properly cited.

\section{INTRODUCTION}

Glass sponges (Hexactinellida: Porifera) provide an abundant source of unusual skeleton structures, which could be defined as natural silica-based nanostructured composite materials. They are intriguing research objects because of the hierarchical organization of their spicules from the nanoscale to the macroscale [1-3]. First observations reported by Lévi et al. [4] on silica-based spicules of a Monorhaphis sponge generated great interest because of their combination of properties, namely, toughness combined with stiffness, and resilience. This sponge species synthesizes the largest biosilica structures on earth [5]. Pencil-sized rod spicules, a meter or more in length, could be bent into a circle without breaking. When the load was released, the spicule recovered its original shape. When the bending of the spicule rod was compared with that of a synthetically derived pure silica rod, the toughness of the spicule was found to be nearly an order of magnitude higher [2]. Recently, the micromechanical properties of biological silica in the giant anchor spicule of Monorhaphis chuni were reported on [6]. Nanoidentation showed a considerably reduced stiffness of the spicule compared to technical quartz glass with different degrees of hydration. Moreover, stiffness and hardness were shown to oscillate as a result of the laminate structure of the spicules. Raman spectroscopic imaging showed that the organic layers are protein-rich and that there is an $\mathrm{OH}$-enrichment in silica near the central axial filament of the spicule. Smallangle X-ray scattering revealed the presence of nanospheres with a diameter of only $2.8 \mathrm{~nm}$ as the basic unit of silica. 
It was suggested that biogenic silica formed by glass sponges possesses reduced stiffness but substantially higher toughness than technical glass due to its architecture, determined by structure at the nanometer and the micrometer level [6]. Unfortunately, the nature and the origin of the protein matrix were not investigated in this study.

There is no doubt that glass sponge anchoring spicules are remarkable objects because of their size, durability, high flexibility, and their exceptional fibre-optic properties, which all together render them of interest as novel biomimetic materials [7]. Of course, the materials science aspects of glass sponges can be studied by model systems, and utilized for biomimetic engineering. However, we cannot mimic nature with a view to designing novel biomaterials without knowledge of the nature and origin of the organic nanostructured matrices of corresponding natural biocomposites which are present in these sponges. Therefore, the biggest shortcoming common to all publications relating to mechanical [2], structural [3], and optical [8] properties of glassy sponge skeletal formations is a lack of real information regarding the chemical nature of corresponding organic matrices.

The finding of collagen within basal spicules of the glass sponge Hyalonema sieboldi [9-11], as well as the occurrence of chitin within the framework skeleton of the glass sponges Farrea occa [12], and spicules of Euplectella aspergillum [7] as revealed by gentle desilicification in alkali, stimulated further attempts to search for materials of organic nature in skeletal structures of these unique deep-sea organisms. Consequently, the objective of the current study was to test our hypothesis that collagen is also an essential component of the giant anchoring silica spicules of Monorhaphis chuni, and if so, to unravel its involvement in the mechanical behavior of these formations, which was well investigated recently [6].

In the present work, we provide a detailed study confirming our hypothesis that the nanofibrillar organic matrix of collagenous nature within the giant spicules of $M$. chuni is responsible for their extraordinary mechanical properties. We performed structural, spectroscopic, and biochemical analyses of these glassy composites. Finally, this work includes a discussion relating to practical applications of silica-collagen composites artificially derived in vitro as biomaterials for use in biomedicine, engineering, and materials science.

\section{EXPERIMENTAL}

\subsection{Chemical etching of spicules and extraction of collagen}

Monorhaphis chuni was collected by the R.V. "Vitiaz-2 (4),"voyage 17 , St. 2601, 12 $2^{\circ} 31.5^{\prime}-25.04^{\prime} S 4^{\circ} 05.5^{\prime}-$ 08.0'E, depth $700 \mathrm{~m}$. Dried Monorhaphis basal spicules (length $120 \mathrm{~cm}$, diameter $1.5-4.5 \mathrm{~mm}$, Figure 1) were washed three times in distilled water, cut into $2-5 \mathrm{~cm}$ long pieces and placed in a solution containing purified Clostridium histolyticum collagenase (Sigma) to digest any possible collagen contamination of exogenous nature. After incubation for 24 hours at $15^{\circ} \mathrm{C}[13]$, the pieces of spicules were washed again three times in distilled water, dried and placed in $10 \mathrm{ml}$ plastic vessels containing $5 \mathrm{ml}$ of $2.5 \mathrm{M} \mathrm{NaOH}$ solution. The

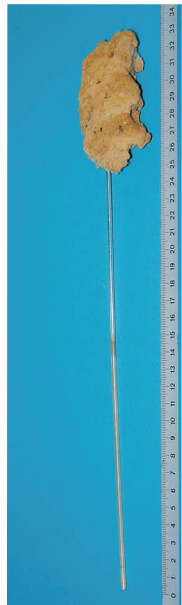

(a)

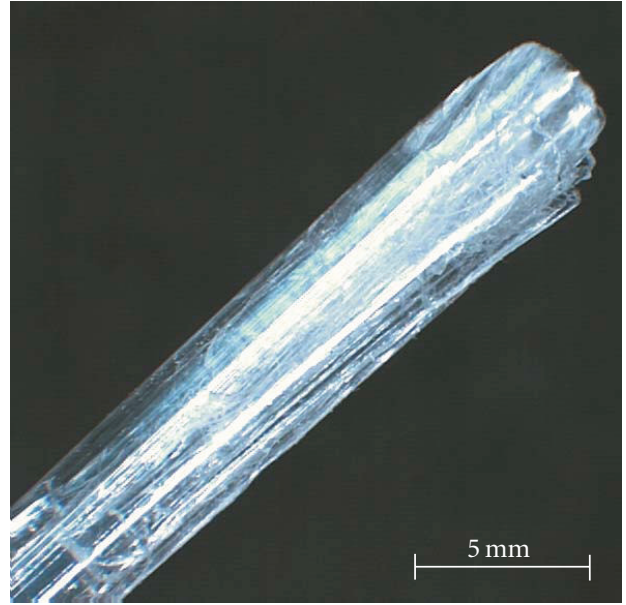

(b)
Figure 1: (a) Marine glass sponge Monorhaphis chuni, a member of the hexactinellids, (b) the sponge consists of a giant basal spicule which anchors Monorhaphis to the sandy substratum.

vessel was covered, placed under thermostatic conditions at $37^{\circ} \mathrm{C}$ and shaken slowly for 14 days. The effectiveness of the slow alkali etching was monitored using scanning electron microscopy (SEM) at different locations along the spicules' length and within the cross-sectional area.

\subsection{Biochemical analysis of collagen}

Alkali extracts of Monorhaphis spicules containing fibrillar protein were dialyzed against deionized water on Roth (Germany) membranes with a cut-off of $14 \mathrm{kDa}$. Dialysis was performed for 48 hours at $4^{\circ} \mathrm{C}$. The dialyzed material was dried under vacuum conditions in a CHRIST lyophilizer (Germany). The approximate molecular weights of proteins in the lyophylizate were determined by gel electrophoresis in the presence of sodium dodecyl sulphate in $10 \%$ and $12 \%$ gel plates. The kit of molecular weight markers (Silver stain SDS molecular standard mixtures) from Sigma, USA, was used. Lyophylizates were dissolved in sample buffer $(1 \mathrm{M}$ Tris- $\mathrm{HCl}$, pH 6.8, 2.5\% SDS, 10\% glycerine, $0.0125 \%$ bromphenol blue) incubated at $95^{\circ} \mathrm{C}$ for 5 minutes and then applied to $10 \%$ or $12 \%$ of SDS-polyacrylamide gels. After electrophoresis at $75 \mathrm{~V}$ for 1.5 hours, $10 \%$ gels were stained with GelCode SilverSNAP Stain Kit II (Pierce,USA), and 12\% gels were stained with coomassie brilliant blue R250 to allow proteins to be visualized. To elucidate the nature of proteins isolated from glass sponge spicules, corresponding electrophoretic gels stained with Coomassie were used for the determination of the aminoacid sequence by the mass spectrometric sequencing technique (MALDI, Finnigan LTQ) as described earlier [14].

\subsection{Structural analysis of spicule layers}

Structural analysis of the glass sponge basal spicules and corresponding extracted proteinaceous components was performed using scanning electron microscopy (SEM) 
(ESEM XL 30, Philips) and transmission electron microscopy (TEM) (Zeiss EM 912). Additional transmission electron microscopy experiments were carried out at the Special Triebenberg Laboratory for electron holography and high-resolution microscopy of the Technical University Dresden.A field-emission microscope of the FEI company (Endhoven, NL) CM200 FEG/ST-Lorentz was used equipped with a $1 \times 1 \mathrm{k}$ CCD camera (multiscan, Gatan, USA). The analysis of the TEM images was realized by means of the Digital Micrograph software (Gatan, USA). Infrared spectra were recorded with a Perkin Elmer FTIR Spectrometer Spectrum 2000, equipped with an AutoImage Microscope using the fourier transform infrared reflection absorption spectroscopy (FT-IRRAS) technique. In the case of the FTIR-analyses, calf skin collagen (Fluka) and Chondrosia reniformis sponge collagen (Klinipharm GmbH, Germany) were investigated as reference samples.

\subsection{Silicification of collagen in vitro}

Tetramethoxysilan (TMOS 98\%, ABCR GmbH, Germany) was chosen as a silica precursor and was hydrolysed for $24 \mathrm{~h}$ at $4^{\circ} \mathrm{C}$ by adding water as well as $\mathrm{HCl}$ as a catalyst. This procedure results in the soluble form of silica-orthosilicic acid-whose further polycondensation reactions can be divided into monomer polymerisation, nuclei growth, and aggregation of particles. Hybridization-the combination of silica and collagen-was performed by intensive mixing of prehydrolysed TMOS and the homogeneous collagen suspensions under ambient conditions as described in [15].

\subsection{Biocompatibility of the silica-collagen hybrid materials}

was evaluated by cultivating human mesenchymal stem cells on the material followed by induced differentiation into osteoblast-like cells [16].

\section{RESULTS AND DISCUSSION}

It was generally accepted that the skeletons of Hexactinellida are composed of amorphous hydrated silica deposited around a proteinaceous axial filament $[17,18]$. The nanolocalization of the proteinaceous component of the glass sponge spicules was not investigated in detail because of lack of a demineralization method which preserved the organic matrix during desilicification. Up to now, the common technique for the desilicification of sponge spicules was based on hydrogen fluoride solutions [5], however this kind of demineralization is rather aggressive chemical procedure which could drastically change the structure of proteins $[19,20]$. To overcome this obstacle, Ehrlich et al. [9-11] developed novel, slow etching methods, which use solutions of $2.5 \mathrm{M} \mathrm{NaOH}$ at $37^{\circ} \mathrm{C}$ and take 14 days. Using these methods, it was shown for the first time that the same class of proteins-collageninvolved in cartilage and bone formation also forms the matrix and deposition site of amorphous silica in $H$. sieboldi glass sponge spicules $[9,21]$. It was suggested that the $H$. sieboldi basal spicule is an example of a biocomposite con- taining a silificated collagen matrix and that the high collagen content is the origin of the high mechanical flexibility of the spicules.

SEM investigations of the alkali-etched Monorhaphis chuni spicules (Figure 2(a)) confirmed the multilayered silica structure, well-known since the first microscopically investigation of hexactinellid sponges by Schultze in 1860 [22], and present in all representatives of lyssacine Hexactinellida [18]. We focused on the investigation of fibrillar components observable at the sites of interstitial layer fractures within partially desilicified spicules. SEM investigations parallel to the slow etching procedures reveal that a fibrillar organic matrix is the template for silica mineralization. Typical fibrillar formations were observed within the tubular silica structures in all layers starting from the inner axial channel containing axial filament (Figure 3(a)) up to the outermost surface layer of the spicules as shown in Figures 2(b) and 2(c). The fibrils in each cylinder form individual concentric $2 \mathrm{D}$ networks with the curvature of the corresponding silicate layers. These layers of about $1 \mu \mathrm{m}$ in thickness are connected among each other by protein fibres (Figure 2(a)), which possess a characteristic nanofibrillar organization (Figures 2(b) and 2(d)). Partially desilicificated nanofibrillar organic matrix observed on the surface of silica-based inner layers of the demineralized spicule provides strong evidence that silica nanoparticles of diameter about $35 \mathrm{~nm}$ are localized on the surface of corresponding nanofibrils (Figures 2(c), 2(e), and Figure 3(b)). This kind of silica nanodistribution is very similar to the silica distribution on the surface of collagen fibrils in the form of nanopearl necklets, firstly observed by us in the glass sponge $H$. sieboldi [21]. We suggest that the nanomorphology of silica on proteinous structures described here could be determined as an example of biodirected epitaxial nanodistribution [23] of the amorphous silica phase on oriented organic fibrillar templates.

The nonsilicificated microfibrils of the M. chuni axial filament with a diameter of approximately $20-30 \mathrm{~nm}$ are organized in bundles with a thickness of $1-2 \mu \mathrm{m}$ oriented along the axis of the spicule. They can be easily identified by SEM (Figure 3(a)). The morphology of these microfibrils observed by TEM (Figures $4(\mathrm{a})$ and $4(\mathrm{~b})$ ) is very similar to nonstriated collagen fibrils isolated previously from $H$. sieboldi [9-11, 21] and examined using electron microscopy.

Except for collagen, there are some other possible candidates (e.g., silicateins of axial filaments such as in Demospongiae $[24,25]$ or as recently reported by Müller et al. $[5,26]$ in $M$. chuni) which would explain the nature and origin of these fibrillar formations. Therefore, a thorough biochemical analysis of isolated fibrils was performed.

The results of the aminoacid analysis of protein extracts isolated from demineralized spicules showed an aminoacid content typical for collagens isolated from several sources listed in Figure 4 and also reported previously [21]. The same extracts were investigated using PAG-electrophoresis. Corresponding electrophoretic gels stained with Coomassie were used for the determination of the aminoacid sequence by a mass spectrometric sequencing technique as described above. We excised two main bands and digested protein material in-gel with trypsin to obtain tryptic peptide mixtures 


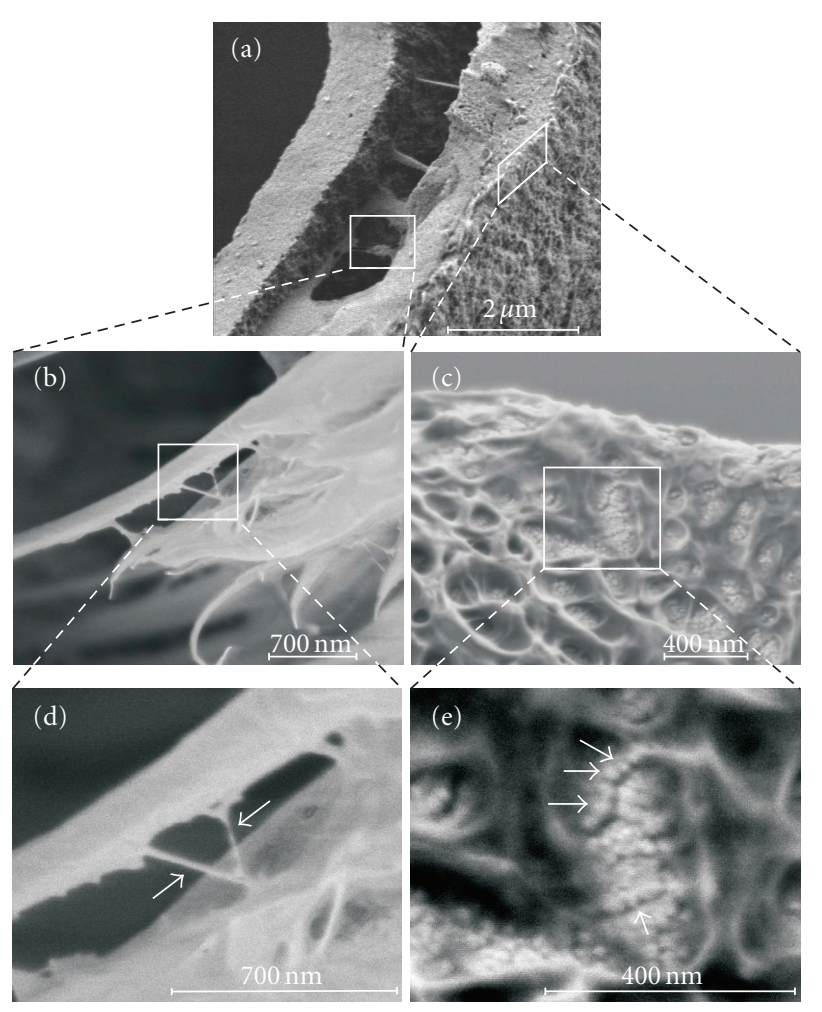

FIGURE 2: SEM images of multilayer constructed $M$. chuni spicule (a) treated with alkali solution which provides strong evidence that the multifibrillar organic matrix is the template for silica mineralization (b)-(e). Spicule layers are connected among each other by nanostructured protein fibres (arrows) (b), (d). Micrograph (e) shows a silica distribution on the surface of nanofibrils in the form of nanopearl necklets (arrows).

for further analysis using LTQ and MALDI peptide finger printings.A comparison to the MSDB protein database [27] led to the identification of collagen alpha 1 in two high MW bands.In contrast to $H$. sieboldi [9], collagen isolated and identified by the same way from Monorhaphis sp. was matched only to type I collagen pre-pro-alpha (I) chain (COL1A1) from dog (AAD34619) (MW 139,74). To our best knowledge, this work is the first study which confirms the presence of collagen within the spicules of Monorhaphis sponge and not only on their surface in the form of a collagen net which covers spicules as recently described by Müller et al. [5].

We also used highly sensitive FTIR methods for the identification of collagen isolated from spicules of $M$. chuni. Spectra obtained from this collagen, calf skin collagen type I and C. reniformis collagen standards were compared to each other in order to elucidate changes in protein secondary structure. The results obtained from the FTIR study (data not shown) show that collagen derived from this glass sponge exhibited spectra very similar to those from calf skin and C. reniformis collagens [28]. The presence of collagen fibrils in alkali solution is no surprise. Hattori et al. [29] investigated the resistance of collagen to alkali treatment at a concentration range of between 3 and $4 \% \mathrm{NaOH}$ at $37^{\circ} \mathrm{C}$ in vitro. The results ob-
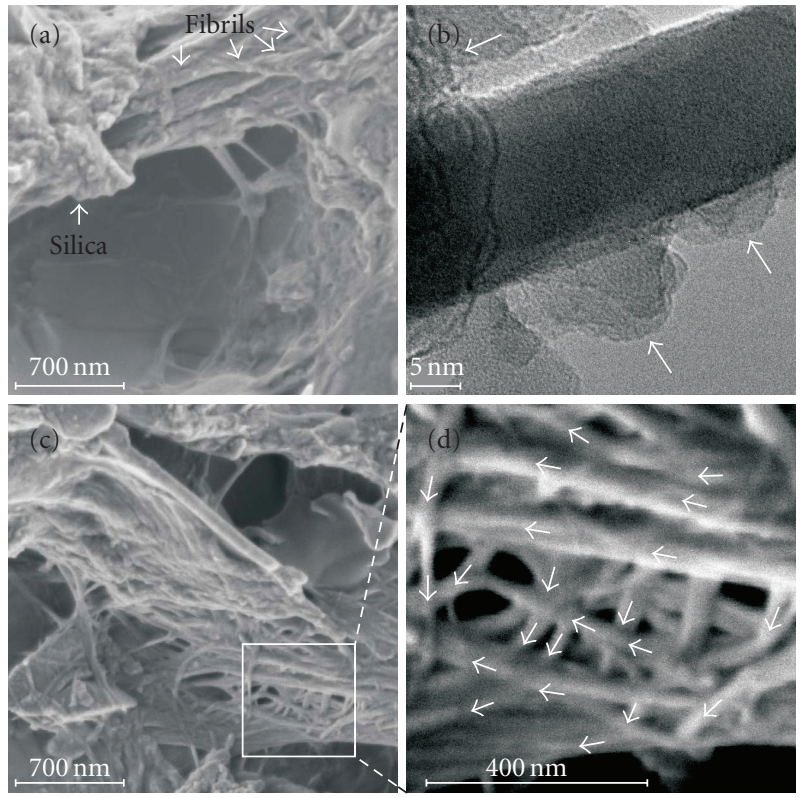

FIGURE 3: SEM and TEM nanoimagery of the fibrillar organic matrix within partially demineralized spicule. (a) Axial filament is an organization of microfibrils with a diameter of approximately 25$30 \mathrm{~nm}$ covered with a silica-containing layer and distributed along the axis of spicule. (b) Nanolocalization of amorphous silica particles (arrows) on the surface of partially demineralized protein fibrils using HRTEM. (c), (d) Collagen fibrils' orientation within spicule possesses a twisted plywood architecture (arrows).

tained indicated that the triple helical conformation and the helicity of the collagen molecule were maintained throughout the period of the alkaline treatment.

The procedure of alkali slow etching opens the possibility to observe the forms of collagen fibrils located within silica layers of spicules and their distribution. The results obtained by SEM observations of the desilicified spicular layers provide strong evidence that collagen fibrils' orientation within $M$. chuni spicules possesses twisted plywood architecture (Figures 3(c) and 3(d)). The twisted plywood or helicoidal structure of collagen fibrils is well-described by Giraud-Guille [30] for bothin vivo and in vitro [31] systems. Spiral twisting of the collagen fibril orientation was found in several biological tissues and described for different organisms including cuticular collagens of polychaete, vestimentifera, scale collagens of primitive and bony fishes, and finally collagen fibers inside bone (all reviewed in [21]).

According to the model proposed by Giraud-Guille, adjacent lamellae have different orientations; either longitudinal (with the collagen fibers along the long axis of the lamellar sheet) or transverse (with the collagen fibers perpendicular to the long axis). From a mechanical point of view, helicoidal structures have certain advantages in resisting mechanical loads compared to orthogonal plywood structures since the twisted orientation enables a higher extensibility in tension and compression [32]. The twisted plywood architecture of collagen fibrils within basal spicules of Monorhaphis visible after alkali treatment (Figure 3) is very 


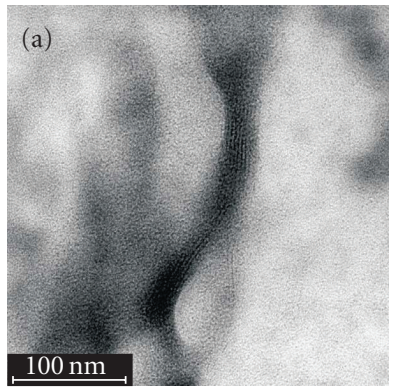

\begin{tabular}{cccc}
\hline $\begin{array}{c}\text { Amino } \\
\text { acid }\end{array}$ & $\begin{array}{c}\text { Monorhaphis } \\
\text { chuni collagen }\end{array}$ & $\begin{array}{c}\text { Hyalonema } \\
\text { collagen [21] }\end{array}$ & $\begin{array}{c}\text { Chondrosia } \\
\text { collagen [21] }\end{array}$ \\
\hline Ala & 6.1 & 6.2 & 6.2 \\
Arg & 4.9 & 4.8 & 4.6 \\
Asx & 10 & 10.7 & 10.4 \\
Cys & 0.1 & 0.2 & 0 \\
Glx & 9.5 & 9.3 & 9 \\
Gly & 25.5 & 24.5 & 30.6 \\
His & 1.4 & 1.6 & 0.4 \\
H-LPro & 7.1 & 6.9 & 9.8 \\
Ile & 3.2 & 3.8 & 2.5 \\
Leu & 4 & 4.3 & 3.6 \\
L-HLys & 1.6 & 1.5 & 1.6 \\
Lys & 2.5 & 2 & 0.7 \\
Met & 0.4 & 0.4 & 0.2 \\
Phe & 2 & 2.2 & 1.8 \\
Pro & 6.7 & 6.5 & 6.3 \\
Ser & 4.4 & 4.7 & 4.5 \\
Thr & 5.2 & 5.6 & 4.3 \\
Tyr & 1.2 & 1.2 & 0.6 \\
Val & 4.8 & 4.1 & 2.9 \\
\hline
\end{tabular}

FIGURE 4: (a) High-resolution transmission electron microscopy image of the fragment of $M$. chuni collagen microfibril; (b) the arrows indicate the presence of nanofibrillar structures with a diameter which corresponds to that of collagen triple helices (1.5 nm). The results of aminoacid analysis (right) of these microfibrills showed an aminoacid content typical for collagens isolated from different sources [21].

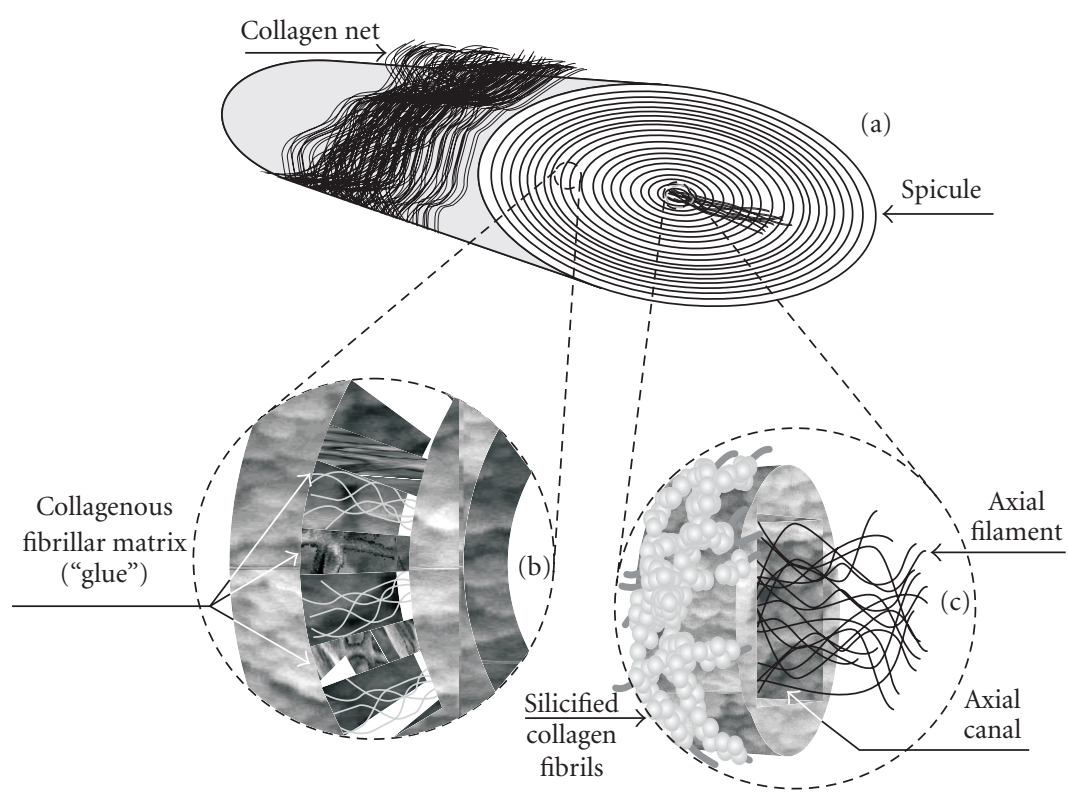

FIGURE 5: proposed model of micro- and nanostructural organisation of the basal spicule of M. chuni with respect to the organic matrix. (a) Collagen nets, surrounding the spicules, showed a tight mat of nanofibrils. Schematic view (b) shows a collagenous fibrillar matrix which could function as a glue between concentric layers. Image (c) represents the region of the axial canal and axial filament. The axial canal of $M$. chuni possesses a characteristic quadratic opening (c) and contains oriented bundles of unsilicified collagenous nanofibrils. The base material of the walls of the axial canal and concentric layers distributed above it consists of silicified collagen fibrils with a twisted plywood orientation. This kind of fibrillar architecture could be responsible for the remarkable micromechanical properties of the spicule as a biocomposite.

similar to that reported for lamellar bone and thus could also confirm the Girauld-Guille model in the case of biosilification in vivo. Correspondingly, this kind of collagen fibril orientation could explain why sponge spicules exhibit specific flexibility and can be bent even to a circle as reported previously $[2,4,21]$. From this point of view, basal spicules of Monorhaphis sponges could be also defined as natural plywood-like silica-ceramics organized similarly to the crossed-lamellar layers of seashells [33]. Thus, we suggest that the matrix of the M. chuni anchoring spicule is silificated fibrillar collagen rather than collagen-containing silica which is the reason for their remarkable mechanical flexibility. 


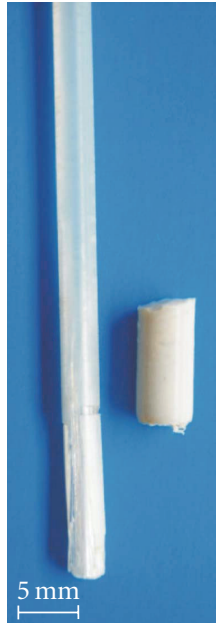

(a)

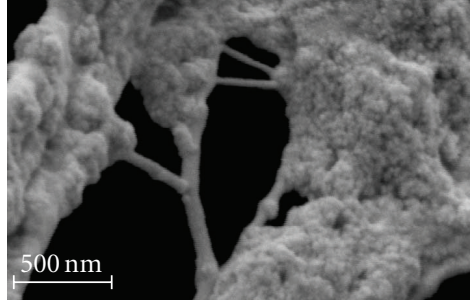

(b)

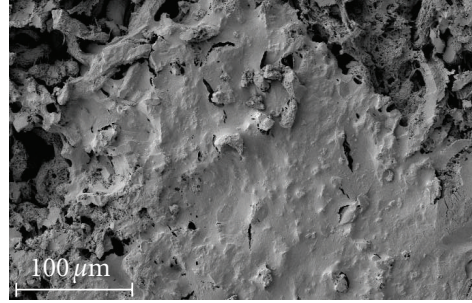

(c)

FIGURE 6: Rod-like collagen-silica-based biomaterial derived in vitro (a) shows morphological similarity to M. chuni basal spicule (a, left). SEM image (b): nanoparticles of amorphous silica deposited in vitro from silicic acid solution on sponge collagen fibrils replicate the nanostructure of glass sponge spicules (Figure 2(e)). SEM micrograph (c) of the surface of silica-collagen hybrid material after 14 days of cultivation of human mesenchymal stem cells, which shows high biocompatibility on this substrate.

Contrary to the postulate that silicateins, as the major biosilica-forming enzymes present in demosponges [34], are responsible for the formation of silica-based structures in all sponges, we suggested that silicateins are associated with collagen [21]. From our point of view, silicateins resemble cathepsins, which are known to be collagenolytic and capable of attacking the triple helix of fibrillar collagens. Therefore, it is not unreasonable to hypothesize that silicateins are proteins responsible for the reconstruction of collagen to form templates necessary for the subsequent silica formation. According to a dynamic model proposed by Müller and his team [5], collagen guides the silicatein(-related) protein/lectin associates concentrically along the spicules of M. chuni. On the basis of the results presented in this paper, we propose a model for the structure of the spicules of Monorhaphis sponges, including micro- and nanoaspects, which can be seen in Figure 5.

Recently, we confirmed that silicification of sponge collagen in vitro occurs via selfassembling, nonenzymatic mechanisms $[15,21]$. To verify whether the collagenous matrix shapes the morphology of the spicules, we carried out invitro experiments in which we exposed collagen to silicic acid solution $\left(\mathrm{Si}(\mathrm{OH})_{4}\right)$. We obtained rod-like structures of several $\mathrm{mm}$ in diameter and demonstrated their similarity to the sponge spicules (Figure 6(a)). The ultrastructural analysis of these selfassembled, collagen-silica composites demonstrates that amorphous silica is deposited on the surface of collagen fibrils in the form of nanopearl necklets (Figure 6(b)), closely resembling the nanoparticulate structure of natural M. chuni spicules (Figures 2(e) and 3(a)).

Bridging the nano- and microlevel, we used different techniques to create a wide spectrum of macroscopic silicacollagen-based hybrid materials. These are highly biocompatible, as demonstrated by the successful cultivation and os- teogenic differentiation of human mesenchymal stem cells on our materials (Figure 6(c)), and potentially useful for technical and biomedical applications. On the basis of the results reported above, we also developed an advanced procedure for the biomimetically inspired production of monolithic silica-collagen hybrid xerogels [16]. The disc-like samples showed convincing homogeneity and mechanical stability, enabling cell culture experiments for the first time on such materials.

\section{CONCLUSION}

Recently, interest in biomaterial properties of silicacontaining structures made by living sponges has grown. In order to exploit the mechanisms for the synthesis of advanced materials and devices, an investigation of the nanoscopic structure of the three-dimensional networks of these remarkable biomaterials needs to be performed [3538]. Understanding the composition, hierarchical structure, and resulting properties of glass sponge spicules gives impetus for the development of equivalents designed in vitro. We showed for the first time that the silica skeletons of hexactinellids represent examples of biological materials in which a collagenous or chitinous organic matrix serves as a scaffold for the deposition of a reinforcing mineral phase in the form of silica. These findings allow us to discard different speculations about materials, which have previously been defined as organic structures (layers, filaments, surfaces) of unknown nature, and open the way for detailed studies on sponge skeletons and spicules as collagen- and/or chitinbased nanostructured biocomposites with high potential for practical applications. 


\section{ACKNOWLEDGMENTS}

This work was partially supported by a joint RussianGerman program "DAAD-Mikhail Lomonosov." We thank Professor H. Lichte for the possibility to use the facilities at the Special Electron Microscopy Laboratory for highresolution and holography at Triebenberg, TU Dresden, Germany. The authors are deeply grateful to Patrice Waridel and Andrei Shevchenko (Max Planck Institute of Molecular Cell Biology and Genetics, Dresden) for the identification of collagen in the composition of spicules, and also to Timothy Douglas, Heike Meissner, Gert Richter, Axel Mensch, and Ortrud Trommer for helpful technical assistance.

\section{REFERENCES}

[1] J. Aizenberg, J. C. Weaver, M. S. Thanawala, V. C. Sundar, D. E. Morse, and P. Fratzl, "Skeleton of Euplectella sp.: structural hierarchy from the nanoscale to the macroscale," Science, vol. 309, no. 5732, pp. 275-278, 2005.

[2] G. Mayer, "Rigid biological systems as models for synthetic composites," Science, vol. 310, no. 5751,pp. 1144-1147, 2005.

[3] J. C. Weaver, J. Aizenberg, G. E. Fantner, et al., "Hierarchical assembly of the siliceous skeletal lattice of the hexactinellid sponge Euplectella aspergillum ," Journal of Structural Biology, vol. 158, no. 1, pp. 93-106, 2007.

[4] C. Lévi, J. L. Barton, C. Guillemet, E. Le Bras, and P. Lehuede, "A remarkably strong natural glassy rod: the anchoring spicule of the Monorhaphis sponge," Journal of Materials Science Letters, vol. 8, no. 3, pp. 337-339, 1989.

[5] W. E. G. Müller, C. Eckert, K. Kropf, et al., "Formation of giant spicules in the deep-sea hexactinellid Monorphaphis chuni (Schulze 1904): electron-microscopic and biochemical studies," Cell and Tissue Research, vol. 329, no. 2, pp. 363-378, 2007.

[6] A. Woesz, J. C. Weaver, M. Kazanci, et al., "Micromechanical properties of biological silica in skeletons of deepsea sponges," Journal of Materials Research, vol. 21, no. 8, pp. 2068-2078, 2006.

[7] H. Ehrlich and H. Worch, "Sponges as natural composites: from biomimetic potential to development of new biomaterials," in Porifera Research: Biodiversity, Innovation \& Sustainability, M. R. Custodio, G. Lobo-Hajdu, E. Hajdu, and G. Muricy, Eds., Museu Nacional, Rio de Janeiro, Brasil, 2007.

[8] J. Aizenberg, V. C. Sundar, A. D. Yablon, J. C. Weaver, and G. Chen, "Biological glass fibers: correlation between optical and structural properties," Proceedings of the National Academy of Sciences of the United States of America, vol. 101, no. 10, pp. 3358-3363, 2004.

[9] H. Ehrlich, T. Hanke, P. Simon, et al., "Demineralization of natural silica based biomaterials: new strategy for the isolation of organic frameworks," BIOmaterialien, vol. 6, no. 4, pp. 297-302, 2005.

[10] H. Ehrlich, T. Hanke, H. Meissner, et al., "Nanoimagery and the biomimetic potential of marine glass sponge Hyalonema sieboldi (Porifera)," VDI Berichte, vol. 1920, pp. 163-166, 2005.

[11] H. Ehrlich, A. V. Ereskovskii, A. L. Drozdov, et al., "A modern approach to demineralization of spicules in glass sponges (Porifera: Hexactinellida) for the purpose of extraction and examination of the protein matrix," Russian Journal of Marine Biology, vol. 32, no. 3, pp. 186-193, 2006.
[12] H. Ehrlich, M. Krautter, T. Hanke, et al., "First evidence of the presence of chitin in skeletons of marine sponges. Part II. Glass sponges (Hexactinellida: Porifera)," Journal of Experimental Zoology Part B, vol. 308, no. 4, pp. 473-483, 2007.

[13] S. Kimura and M. L. Tanzer, "Nereis cuticle collagen. Isolation and properties of a large fragment resistant to proteolysis by bacterial collagenase," Journal of Biological Chemistry, vol. 252, no. 22, pp. 8018-8022, 1977.

[14] A. Shevchenko, M. Wilm, O. Vorm, and M. Mann, "Mass spectrometric sequencing of proteins from silver-stained polyacrylamide gels," Analytical Chemistry, vol. 68, no. 5, pp. 850-858, 1996.

[15] S. Heinemann, H. Ehrlich, C. Knieb, and T. Hanke, "Biomimetically inspired hybrid materials based on silicified collagen," International Journal of Materials Research, vol. 98, no. 7, mbox pp. 603-608, 2007.

[16] S. Heinemann, C. Knieb, H. Ehrlich, et al., "A novel biomimetic hybrid material made of silicified collagen: perspectives for bone replacement," Advanced Engineering Materials, vol. 9, no. 12, pp. 1061-1068, 2007.

[17] J. C. Weaver and D. E. Morse, "Molecular biology of demosponge axial filaments and their roles in biosilicification," $M i$ croscopy Research and Technique, vol. 62, no. 4, pp. 356-367, 2003.

[18] M.-J. Uriz, X. Turon, M. A. Becerro, and G. Agell, "Siliceous spicules and skeleton frameworks in sponges: origin, diversity, ultrastructural patterns, and biological functions," Microscopy Research and Technique, vol. 62, no. 4, pp. 279-299, 2003.

[19] O. Bütschli, "Einige beobachtungen über die kiesel- und kalknadeln von spongien," Zeitschrift für Wissenschaftliche Zoologie, vol. 59, no. 2, mbox pp. 235-286, 1901.

[20] G. Croce, A. Frache, M. Milanesio, et al., "Fiber diffraction study of spicules from marine sponges," Microscopy Research and Technique, vol. 62, no. 4, pp. 378-381, 2003.

[21] H. Ehrlich and H. Worch, "Collagen, a huge matrix in glass-sponge flexible spicules of the meter-long Hyalonema sieboldi," in Handbook of Biomineralization. Vol.1. The Biology of Biominerals Structure Formation, E. Bäuerlein, Ed., Wiley VCH, Weinheim, Germany, 2007.

[22] M. Schultze, Die Hyalonemen. Ein Beitrag zur Naturgeschichte der Spongien, Adolph Marcus, Bonn, Germany, 1860.

[23] T. Kondo, M. Nojiri, Y. Hishikawa, E. Togawa, D. Romanovicz, and R. M. Brown Jr., "Biodirected epitaxial nanodeposition of polymers on oriented macromolecular templates," Proceedings of the National Academy of Sciences of the United States of America, vol. 99, no. 22, pp. 14008-14013, 2002.

[24] J. N. Cha, K. Shimizu, Y. Zhou, et al., "Silicatein filaments and subunits from a marine sponge direct the polymerization of silica and silicones in vitro," Proceedings of the National Academy of Sciences of the United States of America, vol. 96, no. 2, pp. 361-365, 1999.

[25] M. M. Murr and D. E. Morse, "Fractal intermediates in the self-assembly of silicatein filaments," Proceedings of the $\mathrm{Na}$ tional Academy of Sciences of the United States of America, vol. 102, no. 33, pp. 11657-11662, 2005.

[26] X.-H. Wang, J.-H. Li, L. Qiao, et al., "Structure and characteristics of giant spicules of the deep sea hexactinellid sponges of the genus Monorhaphis (Hexactinellida: Amphidiscosida: Monorhaphididae)," Acta Zoologica Sinica, vol. 53, no. 3, pp. 557-569, 2007.

[27] “Sequence Database Setup: MSDB," Imperial College London, http://www.matrixscience.com. 
[28] S. Heinemann, H. Ehrlich, T. Douglas, et al., "Ultrastructural studies on the collagen of the marine sponge Chondrosia reniformis nardo," Biomacromolecules, vol. 8, no. 11, pp. 3452-3457, 2007.

[29] S. Hattori, E. Adachi, T. Ebihara, T. Shirai, I. Someki, and S. Irie, "Alkali-treated collagen retained the triple helical conformation and the ligand activity for the cell adhesion via $\alpha 2 \beta 1$ integrin," Journal of Biochemistry, vol. 125, no. 4, pp. 676-684, 1999.

[30] M. M. Giraud-Guille, "Twisted plywood architecture of collagen fibrils in human compact bone osteons," Calcified Tissue International, vol. 42, no. 3, pp. 167-180, 1988.

[31] M. M. Giraud-Guille, G. Mosser, C. Helary, and D. Eglin, "Bone matrix like assemblies of collagen: from liquid crystals to gels and biomimetic materials," Micron, vol. 36, no. 7-8, pp. 602-608, 2005.

[32] W. Wagermaier, H. S. Gupta, A. Gourrier, et al., "Spiral twisting of fiber orientation inside bone lamellae," Biointerphases, vol. 1, no. 1, pp. 1-5, 2006.

[33] B. Pokroy and E. Zolotoyabko, "Microstructure of natural plywood-like ceramics: a study by high-resolution electron microscopy and energy-variable X-ray diffraction," Journal of Materials Chemistry, vol. 13, no. 4, pp. 682-688, 2003.

[34] H. C. Schröder, D. Brandt, U. Schloßmacher, et al., "Enzymatic production of biosilica glass using enzymes from sponges: basic aspects and application in nanobiotechnology (material sciences and medicine)," Naturwissenschaften, vol. 94, no. 5, pp. 339-359, 2007.

[35] C. W. P. Foo, J. Huang, and D. L. Kaplan, "Lessons from seashells: silica mineralization via protein templating," Trends in Biotechnology, vol. 22, no. 11, pp. 577-585, 2004.

[36] C. Sanchez, H. Arribart, and M. M. Giraud-Guille, "Biomimetism and bioinspiration as tools for the design of innovative materials and systems," Nature Materials, vol. 4, no. 4, pp. 277-288, 2005.

[37] E. Pouget, E. Dujardin, A. Cavalier, et al., "Hierarchical architectures by synergy between dynamical template selfassembly and biomineralization," Nature Materials, vol. 6, no. 6, pp. 434-439, 2007.

[38] P. Fratzl, "Biomimetic materials research: what can we really learn from nature's structural materials?" Journal of the Royal Society Interface, vol. 4, no. 15, pp. 637-642, 2007. 

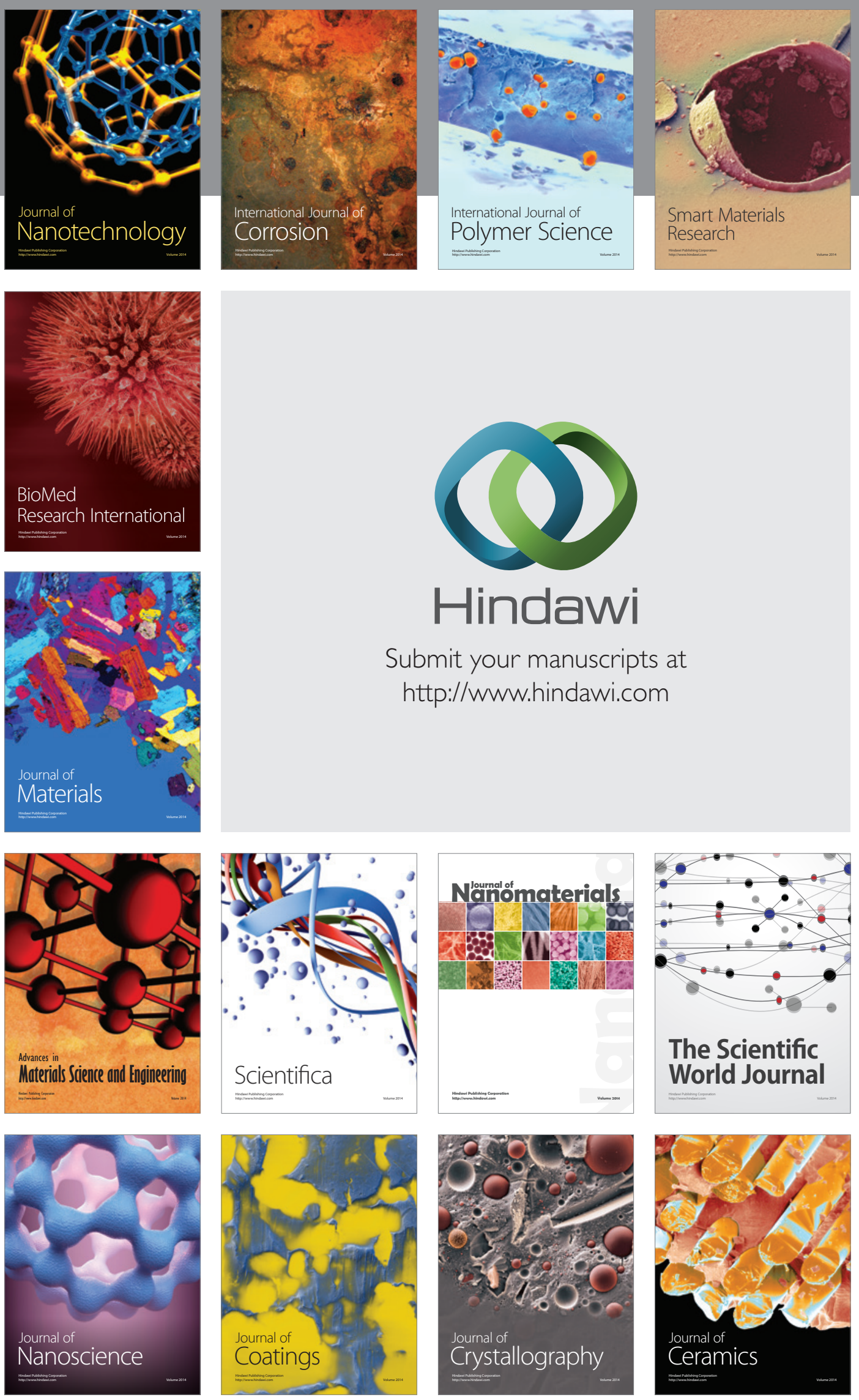

The Scientific World Journal

Submit your manuscripts at

http://www.hindawi.com

\section{World Journal}

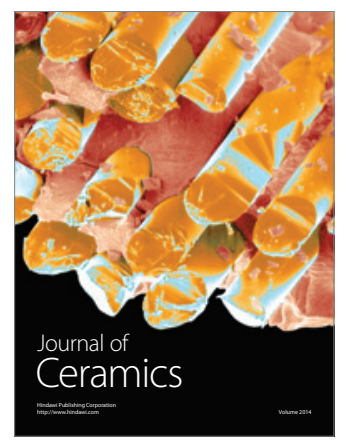

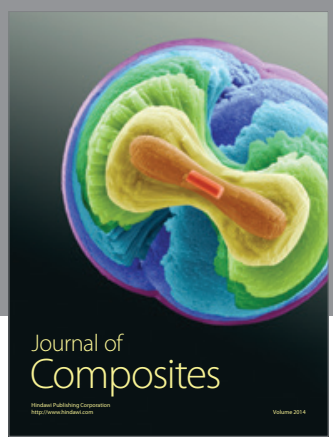
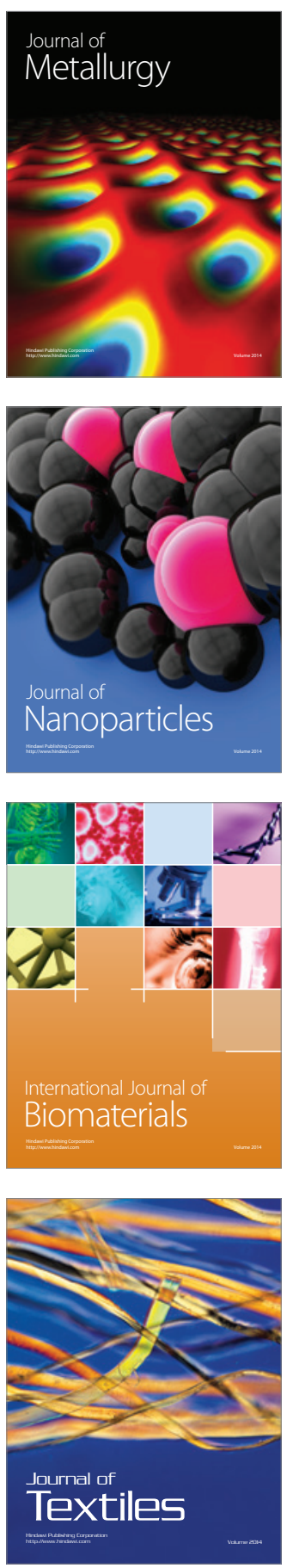